\title{
The association of the FMRFamide-related peptide family with the heart of the stick insect, Baculum extradentatum
}

This article was published in the following Dove Press journal:

Open Access Insect Physiology

15 March 2010

Number of times this article has been viewed

\author{
Amanda Calvin \\ Angela B Lange \\ Department of Biology, University \\ of Toronto Mississauga, \\ Mississauga, ON, Canada
}

\begin{abstract}
The neural anatomy of the heart of the Vietnamese stick insect, Baculum extradentatum consists of a dorsal vessel with lateral cardiac nerves containing cardiac neurons. It also has nerve projections from the segmental nerves. Some of the nerve processes pass over the alary muscles. Multiple pairs of bipolar neurons with axons in the segmental nerve lie on the dorsal diaphragm either side of the heart. FMRFamide-like immunoreactive staining is present in the peripheral nervous system, including the nerve branches from the segmental nerve that project to the heart. Immunoreactive processes are present over the incurrent and excurrent ostia of the heart and in the lateral cardiac nerves, cardiac neurons and in the paired bipolar neurons. SchistoFLRFamide, a myosuppressin, has a dose-dependent inhibitory effect on dorsal vessel contraction. The minimum sequence required for inhibition was examined using truncated peptides, and found to be HVFLRFamide. The truncated peptide VFLRFamide showed activity reversal, being slightly stimulatory. The nonpeptide, benzethonium chloride, mimicked the effects of SchistoFLRFamide. The localization of FMRFamide-like immunoreactivity within the innervation to the heart, coupled with the cardioinhibitory effect of SchistoFLRFamide, suggests a possible role for this family of peptides as neuromodulators in the cardiac musculature in B. extradentatum.
\end{abstract}

Keywords: dorsal vessel, peptide, immunohistochemistry, SchistoFLRFamide, cardiac neurons, excurrent ostia

\section{Introduction}

Circulatory systems have evolved to move nutrients, gases, wastes, and other biologically important material to and from cells of the body, so that the organism can maintain homeostasis. Circulation in vertebrates, and indeed some invertebrates, such as Crustacea, ${ }^{1}$ is highly controlled, such that blood is rerouted to specific tissues as demand increases. Insects have an open circulatory system with the hemolymph (blood) directly bathing the tissues. Hemolymph enters the so-called dorsal vessel via the abdominally-located heart through incurrent ostia (inflow valves). It is pumped anteriorly through the thoracic aorta before exiting into the head capsule. Hemolymph then flows throughout the body cavity, allowing exchange of nutrients and wastes with tissues, before it again enters the posteriorly-located heart. The relatively high metabolic rate in insects demands efficient circulation, and so, in addition to this main circulatory system (dorsal vessel), insects also have accessory pulsatile organs, or auxiliary hearts, that help with circulation in long appendages, such as the legs, antennae, and wings. ${ }^{2}$ Visceral muscle contraction is also important for appropriate circulation. For example, the ovaries are considered an accessory heart in locusts, in that their movement allows
Correspondence: Angela B Lange Department of Biology, University of Toronto Mississauga, 3359 Mississauga Rd, Mississauga, ON, L5L IC6, Canada Tel + I 905 828-3972

Fax + I 905 828-3792

Email angela.lange@utoronto.ca 
for hemolymph to be moved throughout the body cavity, ${ }^{3}$ and the anterior midgut of Rhodnius prolixus has been shown to aid in the movement of diuretic hormone over the Malpighian tubules. ${ }^{4,5}$ Some insects also possess excurrent ostia (outflow valves), which are present on the heart or aorta, and allow hemolymph to pass out of the dorsal vessel directly into the perivisceral cavity (sinus around the alimentary canal). ${ }^{6}$ The presence of these accessory pulsatile organs, along with the excurrent ostia, presumably allows the circulatory system to be more efficient in transporting such items as nutrients, hormones, and waste products throughout the body cavity to the tissues in need.

Hemolymph is pumped anteriorly (typically) by contraction of the muscles of the heart in insects. Dilation of the chambers of the heart results in diastole and is accomplished by contractions of associated alary muscles resulting in hemolymph entering the heart through incurrent ostia. $^{7-9}$ Contractions of the cardiac muscle, which appear to be intrinsic to the muscle (myogenic), close the incurrent ostia and result in systole. This peristalsis moves hemolymph anteriorly towards the head capsule. The situation is more complicated, however, since the heart is also innervated in some insects, and in others it is only innervated at certain developmental stages. ${ }^{10-12}$ In hemimetabolous insects, such as Periplaneta americana and Carausius morosus, paired lateral cardiac nerve cords with intrinsic cardiac neurons appear to control aspects of heart beat, and the heart also receives projections from central neurons by way of the segmental nerves. ${ }^{13-15}$ In addition, a variety of neurochemicals have been shown to be present in this cardioregulatory neuronal system, and to also modulate heart beat. For example, a number of studies have reported the presence of crustacean cardioactive peptide (CCAP), proctolin, and FMRFamiderelated peptides (FaRPs) with the innervation to the dorsal vessel in insects, and have demonstrated effects on heart beat frequency. ${ }^{16-22}$ In addition, the presence of excurrent ostia suggests another form of microcirculatory control, and if these excurrent ostia are controlled (opened and closed) then microcirculatory pathways can be established. This control is suggested by the recent demonstration that the excurrent ostia in B. extradentatum stains with F-actin, indicating the presence of muscle cells. ${ }^{16,17}$

The present paper examines in more detail the innervation to the dorsal vessel of the Vietnamese stick insect B. extradentatum. and uses immunohistochemistry and bioassay to investigate the possible involvement of the family of FaRPs in the control of circulation. This work is important from a comparative point of view, since there are often "extreme differences" in the physiology of hearts of even closely-related species with regard to anatomy (eg, presence or absence of excurrent ostia) and to their physiological response to neuroactive chemicals. ${ }^{9}$

\section{Materials and methods}

\section{Animals}

Vietnamese stick insects, B. extradentatum, were raised at the University of Toronto Mississauga. They were maintained at $25^{\circ} \mathrm{C}$ under high humidity with 12-hour light/12-hour dark light cycle and were fed ficus and oak leaves.

\section{Chemicals}

SchistoFLRFamide (PDVDHVFLRFamide) was purchased from Peninsula Laboratories (Belmont, CA, USA). Truncated analogs of SchistoFLRFamide (HVFLRFamide, VFLRFamide, and PDVDHVFLRF) were custom synthesized by the Core Facility for Protein and Peptide Chemistry, Kingston, ON. Stocks of $10^{-3} \mathrm{M}$ peptide were made up in double-distilled water and stored as $10 \mu \mathrm{l}$ aliquots at $-20^{\circ} \mathrm{C}$. Benzethonium chloride (BZTC) was purchased from SigmaAldrich (Oakville, ON, Canada) and made up in physiological saline prior to use. Physiological saline (pH 6.6; $15 \mathrm{mM} \mathrm{NaCl}$, $18 \mathrm{mM} \mathrm{KCl}, 7.5 \mathrm{mM} \mathrm{CaCl}_{2}, 2 \mathrm{mM}$ HEPES, $50 \mathrm{mM} \mathrm{MgCl}_{2}$, and $184 \mathrm{mM}$ glucose) was used to make working dilutions of the peptide. Rabbit anti-FMRFamide primary antiserum was purchased from INCSTAR (Stillwater, MN, USA) and sheep Cy3 anti-rabbit secondary antibody was purchased from Sigma-Aldrich (Oakville, ON, Canada).

Neurobiotin Tracer, N, (2-aminoethyl) biotinamide hydrochloride, was purchased from Vector Laboratories (Burlington, ON, Canada) and was made up to yield a 25\% stock solution using double-distilled water, and stored at $4^{\circ} \mathrm{C}$. The stock solution was diluted with saline to a final solution of $1.25 \%$ neurobiotin. Purified mouse immunoglobin (Sigma-Aldrich) stored at $4^{\circ} \mathrm{C}$ was used to visualize the neurobiotin tracer $\mathrm{Cy} 3$-conjugated monoclonal anti-biotin. Janus Green B $(0.05 \%$ diluted with saline) was used to trace the peripheral nerves. ${ }^{23}$

\section{Immunohistochemistry}

The immunohistochemistry procedure was modified from Ejaz and Lange ${ }^{16}$ and performed on instars of $3-4 \mathrm{~cm}$ in length. After dissection to expose the heart and nervous system, the preparations were fixed with $4 \%$ paraformaldehyde made up in Millonig's buffer ( $\mathrm{pH} 7.4 ; 0.13 \mathrm{M} \mathrm{NaH}_{2} \mathrm{PO}_{4} \cdot \mathrm{H}_{2} \mathrm{O}$, $0.1 \mathrm{M} \mathrm{NaOH}, 1.2 \%$ glucose, $0.3 \mathrm{mM} \mathrm{CaCl}_{2}$ ) overnight at 
$4^{\circ} \mathrm{C}$. After washing in phosphate-buffered saline (PBS; $10 \mathrm{mM}$ sodium phosphate, $\mathrm{pH} 7.2$ containing $0.9 \% \mathrm{NaCl}$ ) the tissues were incubated for 1 hour in 4\% Triton-X-100 in PBS containing 2\% bovine serum albumin (BSA) and 10\% normal goat serum (NGS). They were then placed in rabbit anti-FMRFamide antiserum (1:1000 in PBS containing 0.4\% Triton-X-100 and $2 \%$ NGS) on a shaker at $4^{\circ} \mathrm{C}$ for 48 hours. Tissues were then washed extensively with PBS, after which they were placed in the Sheep Cy3-conjugated anti-rabbit (1:600 in PBS containing 10\% NGS) on a shaker at $4^{\circ} \mathrm{C}$ overnight. Preparations were washed repeatedly in PBS and run through a glycerol series and mounted on glass slides in $100 \%$ glycerol. Slides were viewed using a Nikon Optiphot 2 Epifluorescence Microscope (Tokyo, Japan) and images were captured using a Zeiss LSM 510 Confocal Laser Microscope (Carl Zeiss, Jena, Germany).

The primary antiserum was preabsorbed overnight in $10^{-5} \mathrm{M}$ SchistoFLRFamide prior to use in the control experiments. No immunoreactive staining was seen in cells or processes on the heart using the preabsorbed antisera.

\section{Neuronal tracing}

The neuronal tracer, neurobiotin, was used to examine the innervation pattern of processes on the heart, as described previously. ${ }^{24}$ The segmental nerve was cut and placed in a well formed by Vaseline walls and the cut end of the nerve was bathed in distilled water for 5 minutes to allow the nerve ending to swell. The distilled water was then replaced with approximately $5 \mu \mathrm{l}$ of $1.25 \%$ neurobiotin. The preparations were kept in a humid chamber overnight and then washed and fixed in $4 \%$ paraformaldehyde at $4^{\circ} \mathrm{C}$ overnight. After extensive washing in PBS the preparations were then placed in $1 \%$ Triton-X-100 (in PBS) for 1 hour, followed by a 2 hour incubation in 10\% NGS and then placed in 1:400 Cy3-conjugated monoclonal anti-biotin, purified mouse immunoglobulin for 48 hours at $4^{\circ} \mathrm{C}$. After washing in PBS, dehydrating through an ethanol series, the preparations were mounted in glycerol and examined for immunostaining, as described above.

\section{Physiological assay}

The head and the legs of the stick insect (instars of 4-5 cm in length) were removed and a lateral incision was made along the entire length of the insect. The preparation was pinned open using minutien pins, the entire digestive system was removed, and the preparation bathed in saline. An impedance converter set on alternating current (short) (UFI, model 2991, Morro Bay, CA, USA) attached to a $200 \mathrm{~mm}$ flat bed chart recorder (VWR, Mississauga, ON, Canada) was used to measure the frequency of dorsal vessel contractions. The electrodes of the impedance converter were placed on either side of the heart in the 1 st abdominal segment. This semiintact dorsal vessel preparation is still connected to the ventral nerve cord (VNC) via one set of segmental nerves. The VNC was removed in some preparations to produce a dorsal vessel preparation fully isolated from the central nervous system (CNS). Preparations were maintained in $0.5 \mathrm{~mL}$ of saline and after a stable heart rate was observed, varying concentrations of SchistoFLRFamide in $0.5 \mathrm{~mL}$ of saline were added after removing the original saline. The preparation was extensively washed between each dose of the peptide.

\section{Results}

\section{General anatomy and innervation}

The Vietnamese stick insect, $B$. extradentatum, has an open circulatory system with a tubular dorsal vessel that extends along the dorsal midline from just beneath the brain to the 9th abdominal segment, gradually increasing in width toward the posterior of the animal. The anatomy of the dorsal vessel has been previously described, but a short description is included here for clarity of the following sections. The dorsal vessel is composed of an anterior portion, referred to as the aorta, and the posterior portion, referred to as the heart. ${ }^{7,16}$ The heart is enclosed by a layer of connective tissue, the dorsal diaphragm, as well as clusters of pericardial cells. Alary muscles, which attach to the body wall, are present at the intersegmental junctions of the heart. The heart also possesses incurrent ostia (valves), situated between each chamber of the heart, to regulate the inflow of hemolymph, which is propelled by waves of contractions anteriorly through the heart and aorta. The presence of eight pairs of incurrent ostia, found solely in the heart, as well as, a single caudal ostium, allows hemolymph to enter the heart. ${ }^{16}$ In these respects the dorsal vessel (aorta and heart) of $B$. extradentatum is similar to that of the Indian stick insect $C$. morosus described by Opoczynska-sembratowa. ${ }^{15}$ Three pairs of excurrent ostia are also present with one pair in the metathoracic segment as well as the 1 st and 2nd abdominal segments of the dorsal vessel permitting hemolymph to exit the heart directly into the perivisceral cavity. ${ }^{15,16}$

Innervation to the dorsal vessel, confirmed here by neurobiotin tracing, neural tracing using Janus Green dye, and immunohistochemistry (see Ejaz and Lange ${ }^{16}$ ), is also similar to that of C. morosus. ${ }^{15,16,25}$ The dorsal vessel is innervated by branches of the segmental nerves that extend from the 
abdominal ganglia of the $\mathrm{VNC}$, and by the lateral cardiac nerves that contain bipolar cardiac neurons. These cardiac neurons extend bilaterally along the entire length of the dorsal vessel. Branches of the segmental nerve extend to the heart wall, as well as to the alary muscles, and again, as with C. morosus $^{15}$ a network of processes originating from two branches of the lateral cardiac nerve and segmental nerve extend over the excurrent ostia. A meshwork of processes also lies over the incurrent ostia.

The neuronal tracer, neurobiotin, also revealed some previously undescribed bipolar neurons (Figure 1). Filling of one lateral segmental nerve from the 2 nd abdominal ganglion revealed the presence of approximately five pairs of bipolar neurons (11-13 $\mu \mathrm{m}$ in diameter) lying in the posterior part of the heart chamber just anterior to the incurrent ostia
(Figure 1). These pairs lay on the ventral surface of the heart, most likely on the dorsal diaphragm, and their cell bodies lay on either side of the dorsal vessel, meaning that one of each pair lay on the contralateral side of the heart to its segmental nerve in which its axon is located. Filling the other lateral segmental nerve showed another five pairs of bipolar neurons that again lay on either side of the dorsal vessel, for a total of ten pairs of bipolar neurons.

\section{Immunohistochemistry}

Extensive FMRFamide-like immunoreactivity (FLI) is distributed along the peripheral nerves and in the nerve branches projecting to the heart and aorta. The commercially available antiserum recognizes peptides with an extended RFamide $\mathrm{C}$ terminus, and so the immunoreactivity might
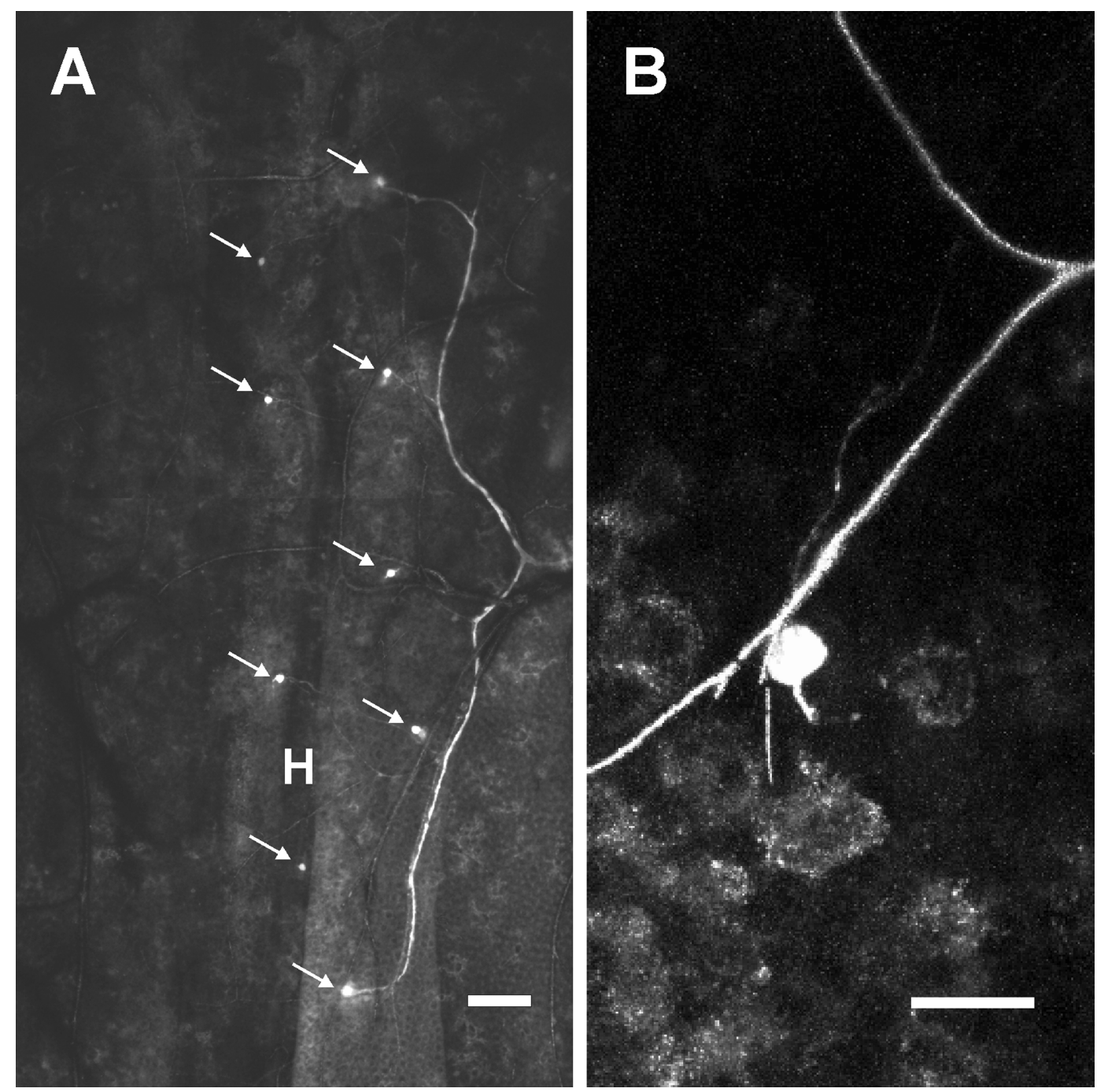

Figure I A) Forward filling of one segmental nerve with the neuronal tracer, neurobiotin, displayed paired bipolar small neurons (arrows) that appeared to lay over the surface of the dorsal diaphragm on either side of the heart $(\mathrm{H})$. B) A magnified view of one of these small neurons filled with neurobiotin.

Notes: Scale bars: A, $100 \mu \mathrm{m}$; B, $25 \mu \mathrm{m}$. 
represent single or multiple members of this extensive FaRP family. The segmental, median, transverse, and link nerves extending from the abdominal ganglia display FLI, with immunoreactive axons extending along the entire length of the branches of the segmental nerves that innervates the heart (Figure 2). Immunoreactive axons continue from the segmental nerves to join the FMRFamide-like immunoreactive lateral cardiac nerves extending the length of the dorsal vessel (Figure 2B). Some of the cardiac neurons stain for FLI, but no link nerve neurons have FLI.
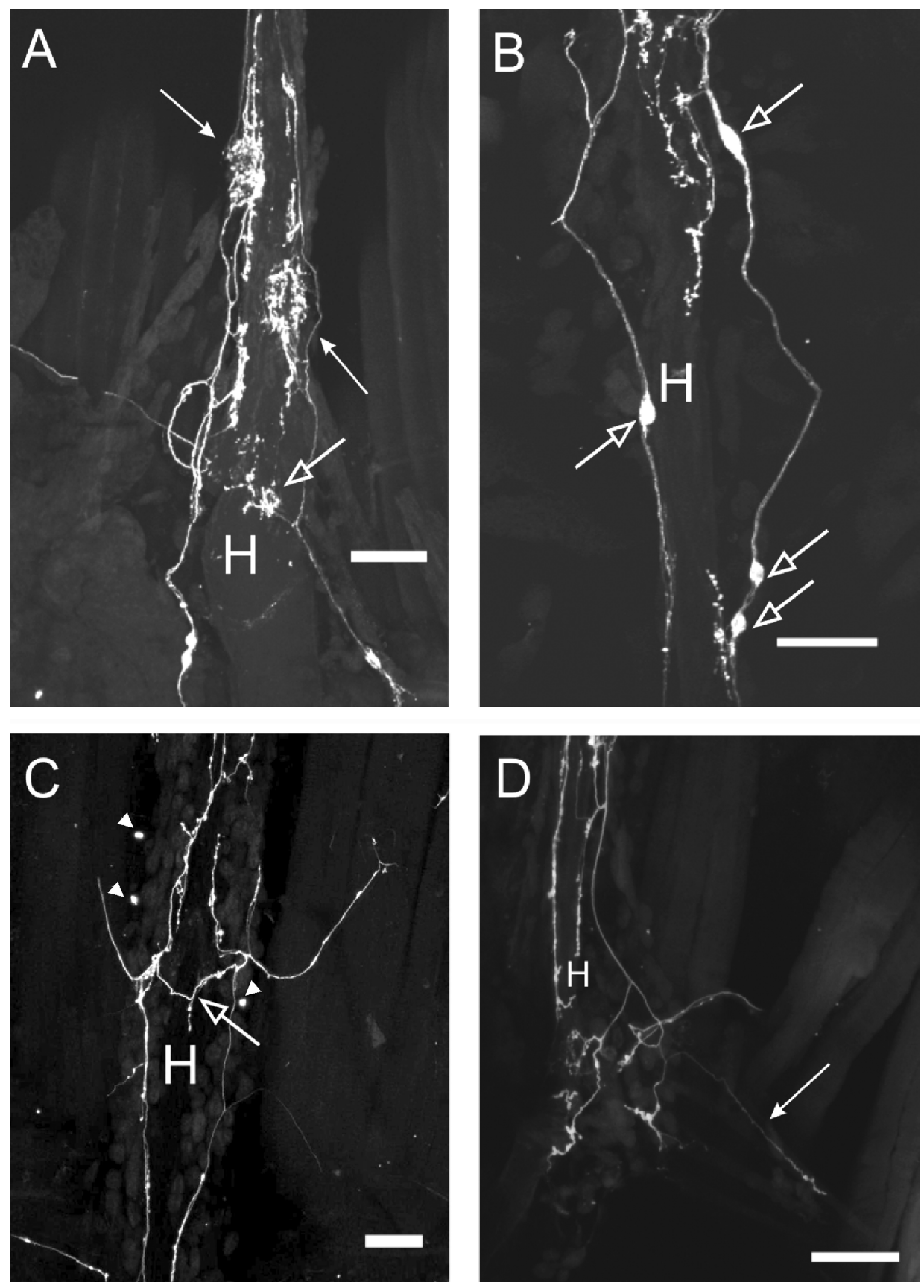

Figure 2 Confocal images of FMRFamide-like immunoreactivity on the heart of B. extradentatum. A) Immunoreactive processes and varicosities extending from axons in the segmental nerve along the heart $(\mathrm{H})$ to the incurrent ostia (open arrow) and anteriorly towards the excurrent ostia (closed arrows) in the Ist abdominal segment. Note that there is a great density of processes around the excurrent ostia. B) FMRFamide-like immunoreactivity in the lateral cardiac nerves and associated cardiac neurons (open arrows) at the junction between the Ist and 2 nd abdominal segment of the heart $(\mathrm{H})$. C) Branch of the segmental nerve displaying FMRFamide-like immunoreactivite processes extending to the incurrent ostia (open arrow) of the heart $(\mathrm{H})$ at the 4th abdominal segment. Note some immunoreactive bipolar neurons lying on either side of the heart (arrowheads). D) An immunoreactive process extending along one alary muscle (closed arrow) of the heart $(\mathrm{H})$ at the 2 nd abdominal segment.

Notes: Scale bar: $100 \mu \mathrm{m}$. 
Each of the three pairs of excurrent ostia are covered with processes of highly intense FLI, with more extensive immunoreactivity associated with the excurrent ostia in the 1st and 2 nd abdominal segments relative to the pair on the aorta (Figure 2A, closed arrows). Staining is also visible in axons branching to the incurrent ostia (Figure 2A, open arrow). The lateral cardiac nerves also possess intense FLI in the region of the excurrent ostia located in the metathoracic to the 3rd abdominal segments of the heart (Figure 2B). The remainder of the lateral cardiac nerves is very much less intense with regard to immunoreactive staining, and indeed, little or no staining is evident on the last two chambers of the heart. Several immunoreactive cardiac neurons $(14 \mu \mathrm{m} \times 24 \mu \mathrm{m})$ of the lateral cardiac nerve are found in the 1 st and 2 nd abdominal segments of the heart (Figure 2B). Several immunoreactive processes and varicosities extend along the heart wall and a few of the alary muscles have processes with FLI (Figure 2).

The bipolar neurons overlying the heart that were revealed with neurobiotin forward filling also stain positively for FLI (Figure 2C). This was confirmed by double-labeling these cells first with neurobiotin forward filling followed by staining for FLI (not shown). Similarly sized bipolar neurons displaying FLI were also seen to be distributed evenly throughout the entire abdomen of the insect, although the axons from which they originate were not traced.

Incubating the anti-FMRFamide antiserum with $10^{-5} \mathrm{M}$ synthetic SchistoFLRFamide eliminated all immunostaining of the dorsal vessel and the peripheral nervous system indicating that the immunostaining was likely due to the presence of FaRPs.

\section{Physiological assay}

The physiological effect of one member of the FaRP family, SchistoFLRFamide, on heart contractions of B. extradentatum was explored by applying varying concentrations of SchistoFLRFamide to the heart, which resulted in a dose-dependent cardioinhibitory effect (Figure 3). The threshold was approximately $10^{-7} \mathrm{M}$ SchistoFLRFamide, which resulted in a decline in frequency of heart contractions of $6.6 \pm 3.7 \%$, and maximal inhibition was at $5 \times 10^{-6}$ M SchistoFLRFamide, decreasing heart rate frequency by $53.6 \pm 7.0 \%$ (Figure 4).

The dorsal vessel was still attached to the CNS via one set of segmental nerves in the above preparations. The dorsal vessel was totally isolated from the CNS to confirm a direct effect of SchistoFLRFamide on the heart muscle. SchistoFLRFamide was still cardioinhibitory in this isolated preparation (data not shown, $\mathrm{n}=4$ ).
There are a variety of FaRPs in insects, and their physiological effects are dependent upon their amino acid sequences. Thus, some truncated versions of SchistoFLRFamide were tested to assess the minimum bioactive sequence. The truncated SchistoFLRFamide, HVFLRFamide, retains its cardioinhibitory activity (Figure 5). However, removal of the His residue, as in VFLRFamide, results in a peptide with no cardioinhibitory activity, and one which has some activity reversal, in that it is slightly cardioexcitatory (Figure 5). Removal of the amide produces a peptide with no biological activity, ie, no effect on heart rate (data not shown, $n=6$ ). The nonpeptide, BZTC, has been shown to be an agonist of the SchistoFLRFamide receptor in locusts ${ }^{26}$ and in a similar manner, BZTC is cardioinhibitory when tested on heartbeat frequency of B. extradentatum (Figure 5).

\section{Discussion}

The anatomy, innervation and function of the dorsal vessel have been examined in a variety of insects. ${ }^{7,9-11,16,17,20,27}$ Miller $^{9}$ discusses the rather "extreme differences" that are evident in these properties of even closely-related species, as well as between distantly-related ones. Thus, in some holometabolous insects, such as Manduca sexta and Drosophila melanogaster the larval heart and aorta lack innervation but at adult metamorphosis innervation appears in distinctive regions. ${ }^{10-12}$ In hemimetabolous insects, such as $P$. americana, C. morosus or Schistocerca gregaria, there is extensive innervation from neurons within the VNC, as well as from intrinsic cardiac neurons of the lateral cardiac nerves. ${ }^{9,15}$ There is also the possibility of neurohormonal regulation in both hemimetabolous and holometabolous insects. The heart and aorta of B. extradentatum is similar to that of C. morosus, and indeed $P$. americana, in that there is extensive innervation from the segmental nerves and intrinsic cardiac neurons of the lateral cardiac nerves. Of particular interest, the excurrent ostia of $B$. extradentatum are targeted with extensively branched nerve processes. Furthermore, a previous study has demonstrated that the excurrent ostia of $B$. extradentatum stain with F-actin, indicating the presence of muscle cells. ${ }^{17}$ This fact, coupled to the meshwork of nerve processes over the excurrent ostia, has led to the suggestion that the excurrent ostia may be neurally-controlled and therefore allow for changes in microcirculation. ${ }^{16,17}$ The incurrent ostia of B. extradentatum are similarly endowed with nerve processes, adding to the further possibility of microcirculatory modulation. Neurobiotin filling of the segmental nerves of $B$. extradentatum also identified numerous paired bipolar neurons located in the posterior half of each chamber of the heart anterior to the 

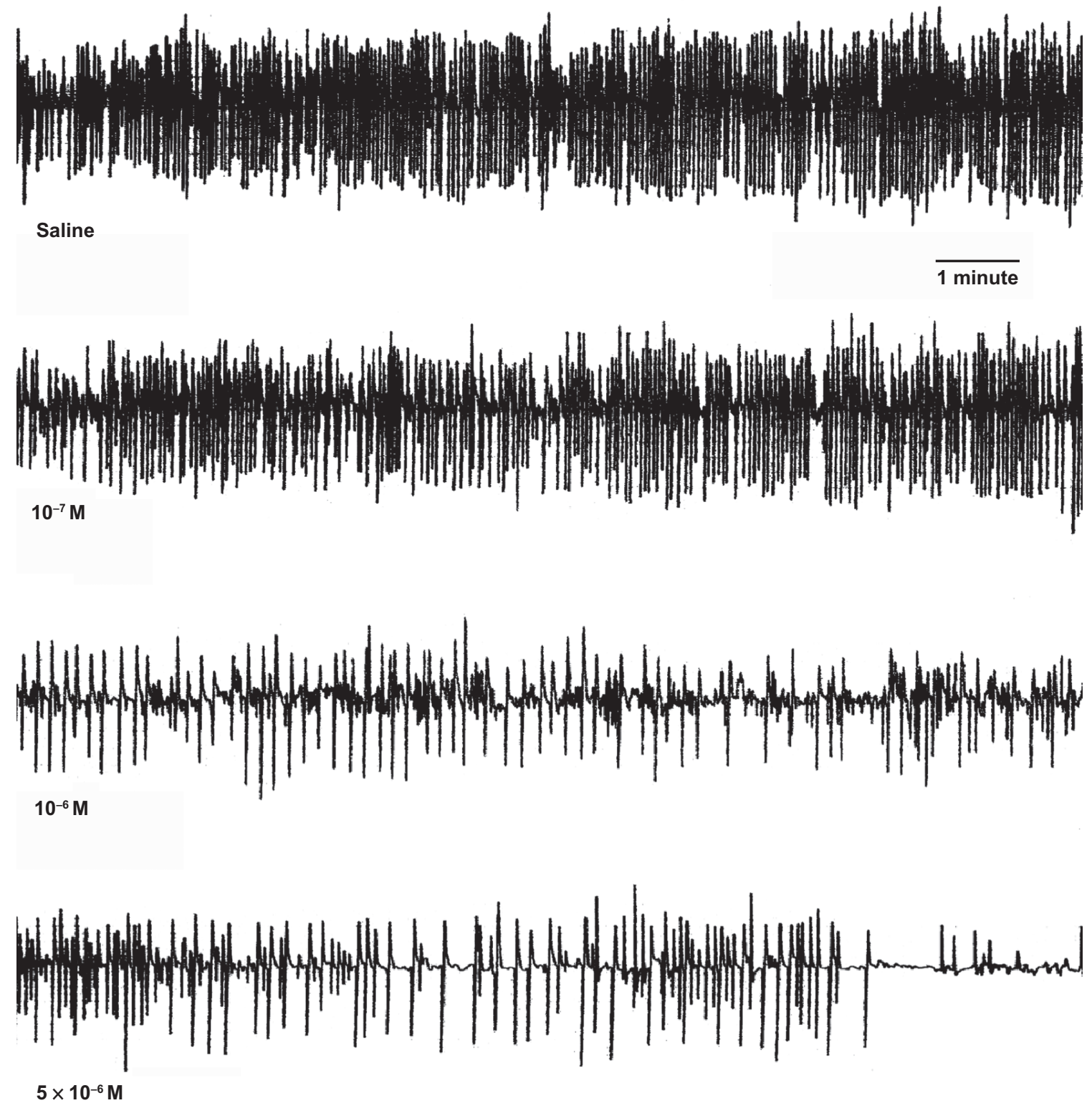

Figure 3 A typical series of sample traces showing the inhibitory effects of SchistoFLRFamide on heart rate in B. extradentatum. Each deflection represents a single contraction of the heart. Trace is taken one minute after the addition of saline or varying concentrations of SchistoFLRFamide (indicated below the trace) to the preparation.

incurrent ostia, with each member of the pair lying on either side of the heart. It is intriguing to suggest that these might be sensory neurons, responding to stretch of the dorsal vessel for feedback purposes, or indeed may be involved in a local reflex monitoring and controlling muscle contractions. Further work is needed to test these hypotheses.

As studied in a variety of insects, immunohistochemistry has demonstrated the association of a variety of neuropeptide families within the innervation to the dorsal vessel and alary muscles. ${ }^{10,16,17,20,28}$ In the current study, FLI was found to be within nerve processes distributed along the dorsal vessel, with several immunoreactive processes and varicosities extending onto the heart, as well as staining of axons in the lateral cardiac nerves. This is consistent with the findings of Miksys and colleagues ${ }^{29}$ for $C$. morosus although interestingly the link nerve neurons of $C$. morosus show FLI, but those in B. extradentatum do not. Highly intense staining was present in nerve processes over the incurrent and excurrent ostia, suggesting again a neural, and peptidergic control over hemolymph entering and exiting the dorsal vessel. Similarly, neurosecretory cells have been found near ostial valves in P. americana and C. morosus, suggestive of the ostia regulating aspects of circulation. ${ }^{30}$ Intensive immunoreactive staining was also visible in and over the segmental nerves projecting to the heart and lateral cardiac nerves that branch along the heart. In addition, the cardiac 

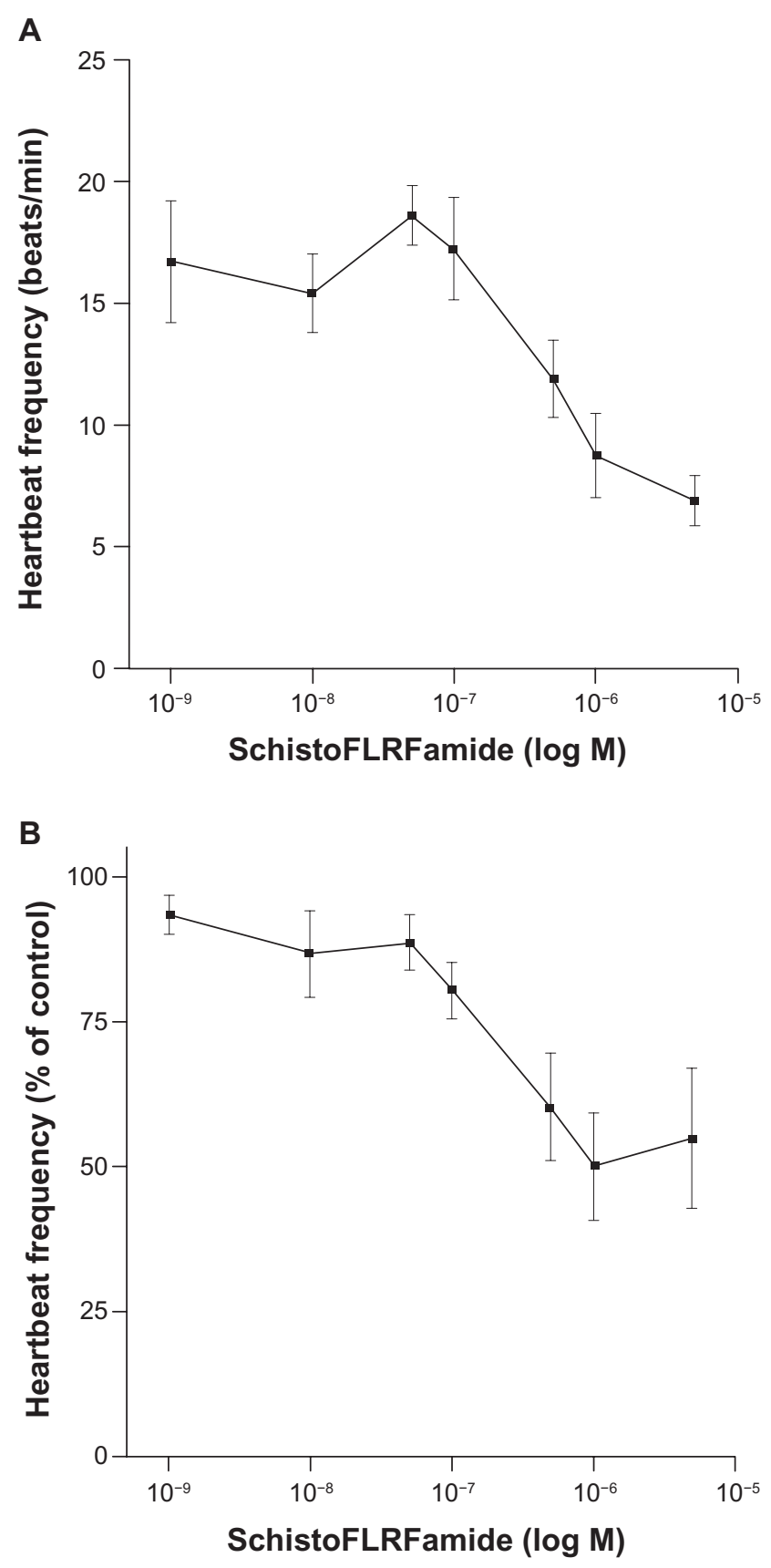

Figure 4 Effect of SchistoFLRFamide on the (A) frequency of spontaneous contractions of the heart (beats/min) and $(\mathbf{B})$ the percentage inhibition of heart rate frequency compared to control (with control as 100\%). The frequency of contraction, between the Ist and 5th minute, was used to determine the average contractions per minute after application. Symbols represent mean \pm SE of 5-9 preparations.

neurons also display FLI although this was limited to the neurons that lie within the first few abdominal segments. Interestingly, the bipolar neurons revealed by neurobiotin fills also possessed FLI. Some sensory neurons in the locust leg are also FMRFamide-like immunoreactive ${ }^{31}$ and so the presence of FLI in the dorsal vessel bipolar neurons does not negate the possibility that they are sensory neurons.
There are various subfamilies of FaRPs in insects ${ }^{32}$ and indeed the commercially available anti-FMRFamide antiserum used in this and other studies stains all members of the FaRPs terminating with RFamide. We chose to test the biological activity of SchistoFLRFamide, since this myosuppressin subfamily has been shown to be associated with the dorsal vessel of other insects. Thus, in S. gregaria, Schoofs and colleagues ${ }^{33}$ found SchistoFLRFamide-like immunoreactivity in axons innervating the heart, and Robb and Evans ${ }^{34}$ discovered SchistoFLRFamide to be inhibitory on $S$. gregaria heart beat frequency. Similarly, Angioy and colleagues ${ }^{27}$ localized dromyosuppressin (DMS)-like immunoreactivity over the heart and anterior aorta of the blowfly, Protophormia terraenovae. They also found DMSlike immunoreactivity in two cells located in the posterior abdominal segment on either side of the midline, with processes projecting to the posterior of the heart. Dromyosuppressin inhibits the fast and slow phases of cardiac activity. In a similar fashion, SchistoFLRFamide inhibits the frequency of heart beat in B. extradentatum in a dose-dependent manner, with a threshold at $10^{-7} \mathrm{M}$ SchistoFLRFamide. In other insect muscle systems, the minimum sequence required to retain the biological activity of SchistoFLRFamide has been shown to be either HVFLRFamide or VFLRFamide. ${ }^{32}$ Thus, we tested these analogs on the $B$. extradentatum heart. The minimum sequence to retain the biological activity of the parent peptide was HVFLRFamide, which is the same pharmacology as shown for the SchistoFLRFamide receptor on locust oviducts, ${ }^{32}$ and for the DMS receptor associated with the slow phase of cardiac activity in $P$. terraenovae. ${ }^{27}$ In addition, the B. extradentatum SchistoFLRFamide-type receptor associated with the dorsal vessel shares other features in common with the oviduct SchistoFLRFamide receptor in that the amide is an absolute requirement for biological activity and the non-peptide BZTC is an agonist. ${ }^{26,32}$

The innervation to $B$. extradentatum dorsal vessel contains other neuropeptides families, including CCAP and procto$\operatorname{lin}^{16,17}$ and these peptides accelerate heart beat frequency. The staining pattern for FLI shares some similarity with the staining pattern for proctolin and CCAP in B. extradentatum, ${ }^{16,18}$ although at present we do not know which members of the FaRP family are actually present. Thus, CCAP-like, proctolin-like and FLI are present within processes over the incurrent ostia and alary muscles. However, differences are also evident, with proctolin-like immunoreactive processes being denser, especially over the alary muscles than for processes with the other peptides, and the excurrent ostia receive processes with FLI and proctolin-like immunoreactivity but 

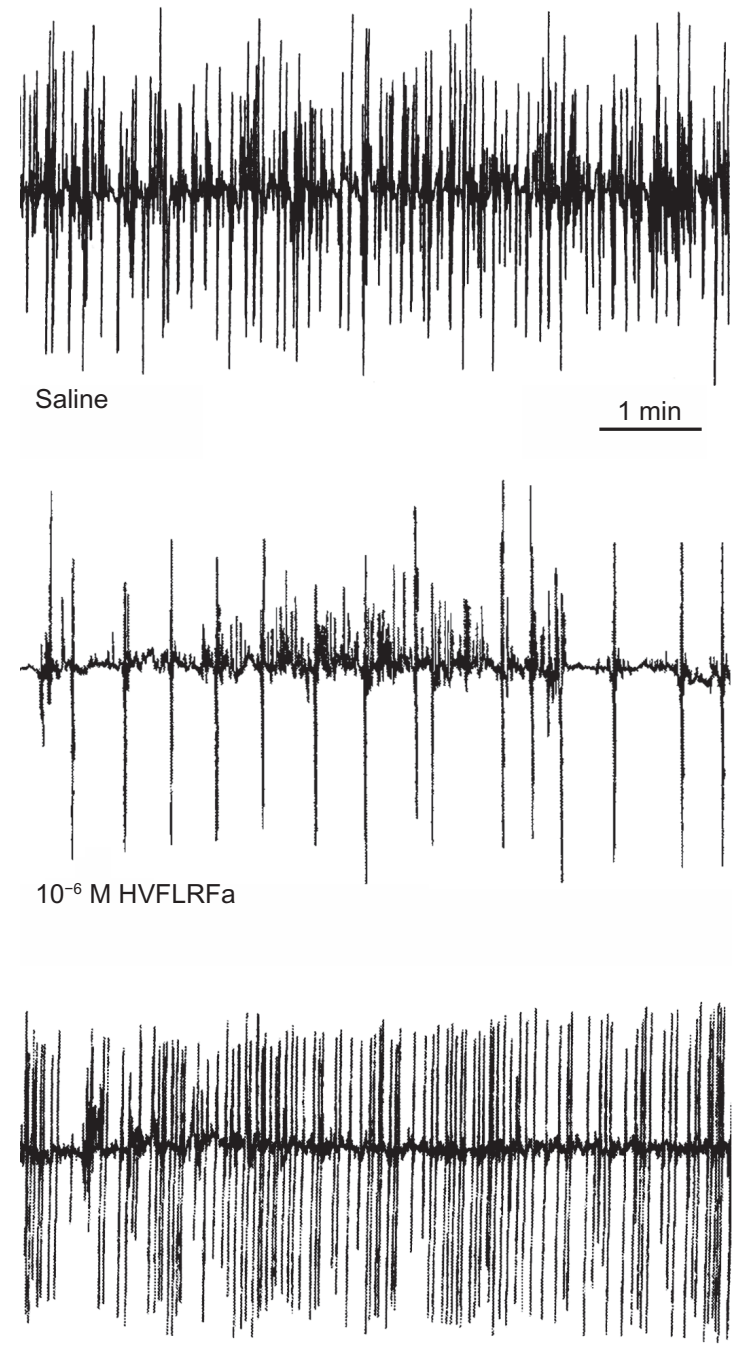

$10^{-6} \mathrm{M}$ VFLRFa

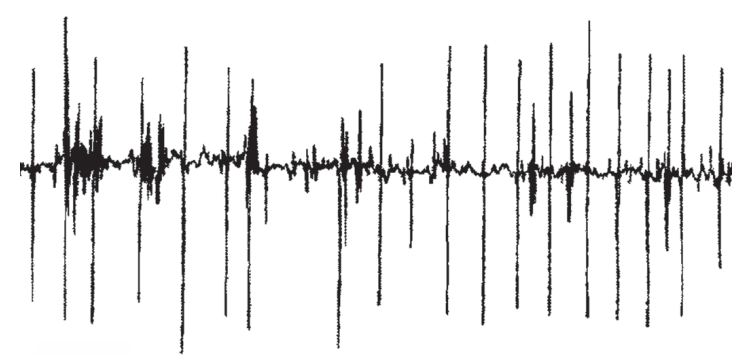

$10^{-6} \mathrm{M} \mathrm{BZTC}$

Figure $\mathbf{5}$ Sample traces of structure-activity studies examining the effects of HVFLRFamide, VFLRFamide and the nonpeptide mimetic benzethonium chloride (BZTC) on heart beat frequency of $B$. extradentatum. $(n=6)$.

not for CCAP-like immunoreactivity. The cardiac neurons stain for FLI but not for proctolin or CCAP. It seems feasible that the neuropeptides associated with the processes over the excurrent ostia may control the opening/closing of the muscular excurrent ostia. Alternatively, or in addition, this may be an appropriate site of release for these neuropeptides as neurohormones, for circulation to distant target tissues. The extensive innervation and differential distribution of neuropeptides along the dorsal vessel of $B$. extradentatum, and their ability to modify heart beat frequency indicates a possible fine control over circulation or microcirculatory control, in this 'open' circulatory system.

\section{Acknowledgments}

This work is supported by Natural Sciences and Engineering Research Council of Canada awards to Amanda Calvin and Angela B Lange. We appreciate the editorial comments of Dr Ian Orchard and some preliminary research done by Mayur Govind. The authors report no conflicts of interest in this work.

\section{References}

1. McMahon BR. Control of cardiovascular function and it evolution in Crustacea. J Exp Biol. 2001;204:923-932.

2. Pass G. Accessory Pulsatile Organs: Evolutionary Innovations in Insects. Annu Rev Entomol. 2000;45:495-518.

3. Orchard I, Lange AB. Peptidergic and aminergic control over an insect visceral muscle. In: 1st International Conference on Insects: Chemical, Physiological and Environmental Aspects, Wroclaw, University of Wroclaw, Poland. 1994. p. 42-50.

4. Maddrell SHP. Excretion in the blood-sucking bug, Rhodnius prolixus Stål. III. The control of the release of the diuretic hormone. J Exp Biol. 1964;41:459-472.

5. Orchard I. Serotonin: A coordinator of feeding-related activities in Rhodnius prolixus. Comp Biochem Physiol. 2006;144:316-324.

6. Nutting WL. A comparative anatomical study of the heart and accessory structures of the orthopteroid insects. J Morphol. 1951;89:501-598.

7. Miller TA. Structure and physiology of the circulatory system. In: Kerkut GA, Gilbert LI, editors. Comprehensive Insect Physiology, Biochemistry and Pharmacology, vol. 3. Oxford, UK: Pergammon Press; 1985:289-353.

8. McCann FV. Physiology of insect hearts. Ann Rev Entomol. 1970;15:173-198.

9. Miller TA. Control of circulation in insects. Gen Pharmac. 1997;29: 23-38.

10. Dulcis D, Levine R. Innervation of the heart of the adult fruit fly, Drosophila melanogaster. J Comp Neurol. 2003;465:560-578.

11. Dulcis D, Levine R. Glutamatergic innervation of the heart initiates retrograde contractions in adult Drosophila melanogaster. J Neurosci. 2005;25:271-280.

12. Davis NT, Dulcis D, Hildebrand JG. Innervation of the heart and aorta of Manduca sexta. J Comp Neurol. 2001;440:245-260.

13. Miller TA. Role of cardiac neurons in the cockroach heartbeat. J Insect Physiol. 1968;14:1265-1275.

14. Alexandrowicz JS. The innervation of the heart of the cockroach (Periplaneta orientalis). J Comp Neurol. 1926;41:291-309.

15. Opoczynska-sembratowa Z. Recherces sur l'anatomie et l'innervation du coeur de Carausius morosus Brunner. Bull Int Acad Sci Letter Cracovie B5. 1936;(7B11):411-436.

16. Ejaz A, Lange AB. Peptidergic control of the heart of the stick insect, Baculum extradentatum. Peptides. 2008;29:214-225.

17. Lange AB, da Silva R. Peptidergic innervation of the excurrent ostia of two Orthopteroid insects. Pestycydy. 2007;3:11-16. 
18. Lange AB, Calvin A, da Silva R. Neuropeptides modulate the heart of the stick insect, Baculum extradentatum. Ann NY Acad Sci. 2008;1163:448-450.

19. Nässel DR. Neuropeptides in the nervous system of Drosophila and other insects: Multiple roles as neuromodulators and neurohormones. Prog Neurobiol. 2002;68:1-84.

20. Dircksen H, Müller A, Keller R. Crustacean cardioactive peptide in the nervous system of the locust, Locusta migratoria: an immunocytochemical study on the ventral nerve cord and peripheral innervation. Cell Tissue Res. 1991;263:439-457.

21. Stevenson PA, Pflüger HJ. Colocalization of octopamine and FMRFamide related peptide in identified heart projecting (DUM) neurons in the locust revealed by immunocytochemistry. Brain Res. 1994;638:117-125.

22. Nichols R. Signalling pathways and physiological functions of Drosophila melanogaster FMRFamide-related peptides. Annu Rev Entomol. 2003;48:485-503.

23. Yack JE. Janus Green B as a rapid, vital stain for peripheral nerves and chordotonal organs in insects. J Neurosci Methods. 1993;49:17-22.

24. Mesce KA, Klukas KA, Brelje TC. Improvements for the anatomical characterization of insect neurons in whole mount: the use of cyaninederived fluorophores and laser scanning confocal microscopy. Cell Tissue Res. 1993;271:381-397.

25. Fifield M, Finlayson LH. Peripheral neurons and peripheral neurosecretion in the stick insect, Carausius morosus. Proc Royal Society Lond Issue B. 1978;200:63-85.

26. Lange AB, Orchard I, Wang Z, Nachman RJ. A nonpetide agonist of the invertebrate receptor for SchistoFLRFamide (PDVDHVFLRFamide), a member of a subfamily of insect FMRFamide-related peptides. Proc Natl Acad Sci U S A. 1995;92:9250-9253.
27. Angioy AM, Muroni P, Barbarossa IT, McCormick J, Nichols R. Evidence dromyosuppressin acts at posterior and anterior pacemakers to decrease the fast and the slow cardiac activity in the blowfly Protophormia terraenovae. Peptides. 2007;28:585-593.

28. Sarkar NRS, Tobe SS, Orchard I. The distribution and effects of Dippu-allatostatin-like peptides in the blood-feeding bug Rhodnius prolixus, Peptides. 2003;24:1553-1562.

29. Miksys S, Lange AB, Orchard I, Wong V. Localization and neurohemal release of FMRFamide-related peptides in the stick insect Carausius morosus. Peptides. 1996;18:27-40.

30. Miller T. Role of cardiac neurons in the cockroach heartbeat. $J$ Insect Physiol. 1968;14:1265-1275.

31. Persson MGS, Nässel DR. Neuropeptides in insect sensory neurones: tachykinin-, FMRFamide- and allatotropin-related peptides in terminals of locust thoracic sensory afferents. Brain Res. 1999;816:131-141.

32. Orchard I, Lange AB, Bendena WG. FMRFamide-related peptides: A multifunctional family of structurally related neuropeptides in insects. Adv Insect Physiol. 2001;28:267-329.

33. Schoofs L, Holman GM, Paemen L, Veelaert D, Amelinckx M, De Loof A. Isolation, identification and synthesis of PDVDHFLRFamide (SchistoFLRFamide) in Locusta migratoria and its association with the male accessory glands, the salivary glands, the heart, and the oviduct. Peptides. 1993;14:409-421.

34. Robb S, Packman LC, Evans PD. Isolation, primary structure and bioactivity of SchistoFLRFamide, a FMRF-amide-like neuropeptide from the locust, Schistocerca gregaria. Biochem Biophysic Res Commun. 1989;160:850-856.
Open Access Insect Physiology

\section{Publish your work in this journal}

Open Access Insect Physiology is an international, peer-reviewed, open access journal publishing original research, reports, reviews and commentaries on all areas of insect physiology. The manuscript management system is completely online and includes a very quick and fair peer-review system, which is all easy to use.

\section{Dovepress}

Visit http://www.dovepress.com/testimonials.php to read real quotes from published authors. 